\title{
Understanding and resolving the discrepancy between differential and actual minority carrier lifetime
}

\author{
J. A. Giesecke, S. W. Glunz, and W. Warta \\ Fraunhofer Institut für Solare Energiesysteme, Heidenhofstr. 2, 79110 Freiburg, Germany
}

(Received 6 November 2012; accepted 24 January 2013; published online 19 February 2013)

\begin{abstract}
Differential light-biased dynamic measurements of charge carrier recombination properties in semiconductors have long been known to yield only differential rather than actual recombination properties. Therefore, the determination of injection-dependent recombination properties from such measurements was previously found to require integration over the entire injection range. Recent investigations of the phase shift between a time-modulated irradiation of silicon samples and excess carrier density reveal a striking analogy to the above findings: the phase shift is greater than the actual effective carrier lifetime in the case of a positive derivative of lifetime with respect to excess carrier density, and vice versa. This work attempts to rearrange the mentioned previous findings in a quantitative theoretical description of light-biased dynamic measurements of effective carrier lifetime. Both light-biased differential lifetime measurements as well as harmonically time-modulated methods without additional bias light are shown to represent a limiting case in a general treatment of light-biased dynamic lifetime measurements derived here. Finally, we sketch a way to obtain actual recombination properties from differential measurements-referred to as a differential-to-actual ( $d 2 a$ ) lifetime analysis, which does not require integration over the entire injection range. (C) 2013 American Institute of Physics. [http://dx.doi.org/10.1063/1.4790716]
\end{abstract}

\section{INTRODUCTION}

Dynamic measurement techniques of charge carrier recombination properties such as effective carrier lifetime feature distinct advantages over steady-state techniques. Most prominently, they measure lifetime directly from the decay of excess carrier density rather than to implicitly infer lifetime from measured steady-state amplitudes via possibly violated assumptions. Yet, several limitations and artifacts of dynamic lifetime techniques have been addressed in the past. ${ }^{1-5}$

In 1995, Brendel, Aberle, and Schmidt identified a discrepancy between actual (true) injection-dependent effective surface recombination velocity and a so-called differential surface recombination velocity inferred from light-biased differential photoconductance decay measurements. ${ }^{1,2}$ Due to the differential nature of the measured quantity, it was concluded that integration of measurements throughout the entire injection range was essential in order to obtain correct injection-dependent surface recombination velocity from differential decay time measurements. ${ }^{2}$

In our recent work on carrier lifetime measurements via time-modulated or quasi-steady-state photoluminescence, we made very similar observations to the findings of Brendel, Aberle, and Schmidt. Our dynamic photoluminescence setup features a sinusoidally time-modulated irradiation without bias light. Following the self-consistent dynamic approach by Trupke et al., ${ }^{6}$ we aimed at overcoming its requirement of assumptions about net dopant concentration under intermediate- to high-level injection conditions. ${ }^{7}$ For this purpose, we initially interpreted the phase shift between time-modulated irradiation and photoluminescence intensity as the actual effective lifetime-as one might conclude from a quasisteady-state treatment of the time-dependent continuity equation. ${ }^{8}$ However, measured phase shifts proved to only be equal to actual effective carrier lifetime in the special case of a vanishing injection dependence. This followed from contradicting self-consistent and phase shift lifetime analyses. Also, numeric modeling of the phase shift between a time-modulated irradiation and excess carrier density revealed systematic deviations from actual lifetime for injection-dependent lifetimes. ${ }^{7,8}$ The phase shift was greater than effective carrier lifetime in the case of a positive derivative of lifetime with respect to excess carrier density $d \tau / d \Delta n>0$, whereas it was less than lifetime in the case of a negative derivative $d \tau / d \Delta n<0$. Strikingly, this is a qualitative analogy to the findings of Brendel, Aberle, and Schmidt. Beyond that, quantitative agreement with the case of differential decay time measurements is elaborated theoretically and substantiated by simulations in this paper.

This work attempts to generalize the findings about the discrepancy between differential and actual recombination properties by Brendel, Aberle, and Schmidt to dynamic measurements of effective carrier lifetime. The mentioned discrepancy is theoretically derived as a function of the irradiation conditions of light-biased dynamic lifetime measurements. Both light-biased differential dynamic lifetime measurements as well as measurements of effective carrier lifetime as inferred from the phase shift between a timemodulated irradiation and free carrier density are shown to represent a most affected limiting case. Dynamic carrier lifetime measurement techniques are classified in terms of whether or not they suffer from the addressed discrepancy. Finally, a generally valid approach (even for nonlinear lifetime) to obtain actual recombination properties from the affected methods without requiring integration over the entire injection range is sketched. 


\section{THEORY}

The essential differential equation to describe charge carrier dynamics in optically irradiated silicon devices is the continuity equation

$$
\frac{\partial \Delta n}{\partial t}-D_{n} \frac{\partial^{2} \Delta n}{\partial z^{2}}+\frac{\Delta n}{\tau_{n}}=G
$$

with the time- and depth-dependent excess carrier density $\Delta n(z, t)$, the generation rate of electron hole pairs $G(z, t)$, the minority carrier bulk lifetime $\tau_{n}$, and the minority carrier diffusion coefficient $D_{n}$. Some previous papers on this topic had focussed on the measurement of surface recombination velocities $S_{f / r},{ }^{1,2}$ which come into play as recombination rates through the boundary conditions at device interfaces at $z=0, d$

$$
\left.D_{n} \frac{\partial \Delta n}{\partial z}\right|_{z=0, d}= \pm\left. S_{f / r} \Delta n\right|_{z=0, d}
$$

Finite positive surface recombination velocities lead to a negative depth integrated contribution of the second derivative of excess carrier density with respect to depth $\partial^{2} \Delta n / \partial z^{2}$ in the continuity equation. Therefore, in a solely timedependent treatment of the continuity equation, the positive term $-D_{n} \partial^{2} \Delta n / \partial z^{2}$ is to be interpreted as a surface recombination rate. With an effective carrier lifetime $\tau$, total recombination rate reads

$$
\frac{\Delta n(t)}{\tau}=\frac{1}{d} \int_{0}^{d} d z\left(\frac{\Delta n(z, t)}{\tau_{n}}-D_{n} \frac{\partial^{2} \Delta n(z, t)}{\partial z^{2}}\right),
$$

and the resulting ordinary differential equation (with depthaveraged generation rate and excess carrier density) reads

$$
\frac{d \Delta n}{d t}+\frac{\Delta n}{\tau}=G
$$

This simplification implies no loss of generality with regard to the conclusions drawn in this paper. Aberle et al. already pointed out that their derivation for surface recombination velocities was transferrable to bulk recombination. ${ }^{2}$ The same applies to effective lifetime, being representative of the sum of surface and bulk recombination rates-as already addressed by both Schuurmans et al. ${ }^{3}$ and Schmidt. ${ }^{4}$

In the following, two questions are addressed: The first is concerned with the structural change imposed on the continuity equation through the injection dependence of lifetime, and whether it could affect the determination of lifetime from decay time measurements. Second, the impact of a light bias on the determination of lifetime from decay time measurements is systematically elaborated.

\section{A. Structural change of the continuity equation}

One might argue that the continuity equation encounters a structural change through the injection dependence of lifetime $\tau(\Delta n)$. In order to investigate the impact of this structural change on measurable decay time, a first order linear series expansion of the continuity equation in $\Delta n$ was solved for an abruptly vanishing steady-state generation rate $G(t \leq 0)=G_{0}=\Delta n_{0} / \tau_{0}$ and $G(t>0)=0$. With an injection-dependent lifetime

$$
\tau(\Delta n) \approx \tau_{0}+\left.\left(\Delta n-\Delta n_{0}\right) \frac{d \tau}{d \Delta n}\right|_{\Delta n_{0}}
$$

for very small times $t>0$ and $\Delta n(t=0)=\Delta n_{0}$ the linearized continuity equation takes the form

$$
\frac{d \Delta n}{d t}+U \Delta n=V
$$

with coefficients

$$
\begin{aligned}
& U=\frac{1-\left.G_{0} \frac{d \tau}{d \Delta n}\right|_{\Delta n_{0}}}{\tau_{0}}, \\
& V=-\left.G_{0}^{2} \frac{d \tau}{d \Delta n}\right|_{\Delta n_{0}} .
\end{aligned}
$$

The solution of Eq. (6) for very small times $t>0$ is

$$
\Delta n(t)=\left(\Delta n_{0}-\frac{V}{U}\right) \exp (-U t)+\frac{V}{U} .
$$

Evaluation of measurable decay time $\tau_{m}(t \rightarrow 0)$ then yields

$$
\tau_{m}(t \rightarrow 0)=-\left.\frac{\Delta n}{d \Delta n / d t}\right|_{t \rightarrow 0}=\frac{-\Delta n_{0}}{-U \Delta n_{0}+V}=\tau_{0} .
$$

Thus, one may conclude that the mismatch between differential decay time $\tau_{m}$ and actual lifetime $\tau$ is not solely caused by the structural change of the continuity equation through the injection dependence of lifetime. As will be derived in the following, a mismatch occurs only if injection-dependence of carrier lifetime is accompanied by a light bias.

\section{B. Theory of light-biased decay time}

The following derivation is concerned with the measurable decay time $\tau_{m}(t \rightarrow 0)$ after an abrupt change of generation rate $G(t \leq 0)=G_{0}=\Delta n_{0} / \tau_{0}$ and $G(t>0)=\xi G_{0}$ with a bias light parameter $0 \leq \xi<1$. According to Eq. (4), evaluation of the derivative of the solution of the continuity equation with respect to time at very small times $t>0$ then yields

$$
\frac{d \Delta n}{d t}(t \rightarrow 0)=-G_{0} \cdot(1-\xi) .
$$

In the calculation of the measurable decay time $\tau_{m}(t \rightarrow 0)$, the fact that excess carrier density decays to an equilibrium value $\Delta n(t \rightarrow \infty) \neq 0$ has to be accounted for. Thus, it must read

$$
\tau_{m}(t \rightarrow 0)=\frac{\Delta n_{0}-\Delta n(t \rightarrow \infty)}{(1-\xi) G_{0}} .
$$


This form reveals the differential nature of the measurable decay time $\tau_{m}(t \rightarrow 0)$, as it relates a change of generation rate to a change of excess carrier density. The equilibrium value $\Delta n(t \rightarrow \infty)$ is affected by the injection dependence of lifetime. As for $t \rightarrow \infty$ the derivative of excess carrier density with respect to time must vanish, combination of Eq. (4) with the injection-dependent lifetime (cf. first order Taylor expansion given by Eq. (5)) leads to

$$
\Delta n(t \rightarrow \infty)=\xi G_{0} \cdot\left(\tau_{0}+\left.\left(\Delta n(t \rightarrow \infty)-\Delta n_{0}\right) \frac{d \tau}{d \Delta n}\right|_{\Delta n_{0}}\right)
$$

Solving this equation for $\Delta n(t \rightarrow \infty)$, one obtains

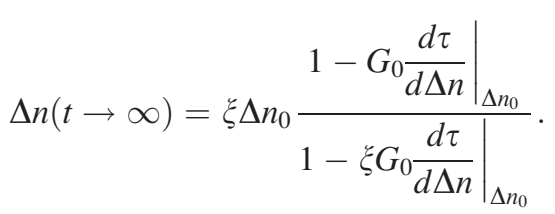

It can be shown (cf. Appendix) that this expression is generally valid in the limit $\xi \rightarrow 1$, even when accounting for higher order Taylor expansions of nonlinear injectiondependent carrier lifetime in Eq. (12). Insertion into Eq. (11) then leads to the resulting expression

$$
\tau_{m}(t \rightarrow 0)=\frac{\tau_{0}}{1-\left.\xi G_{0} \frac{d \tau}{d \Delta n}\right|_{\Delta n_{0}}}
$$

Due to the linear approximation of lifetime in Eq. (12), Eq. (14) is also an approximation. However, in the limit $\xi \rightarrow 1$ it is exact in any case, because of the general validity of Eq. (13) in this limit (cf. Appendix). Note that we thoroughly checked the exact validity of Eq. (14) for nonlinear $\tau(\Delta n)$ in the limit $\xi \rightarrow 1$ via numerical simulations (cf. Sec. IV, Fig. 6). For finite differences between $\xi$ and unity, the accuracy of Eq. (14) depends on the linearity of $\tau(\Delta n)$ in the proximity of $\Delta n_{0}$. In most cases, it is deemed very accurate for values $\xi>0.9$. For $\xi=0$, it follows directly from Eq. (11) that $\tau_{m}(t \rightarrow 0)=\tau_{0}$.

\section{IMPLICATIONS OF LIGHT-BIASED DECAY THEORY}

Equation (14) is essential for the theory of light-biased decay time derived herein. It provides a quantitative measure of the discrepancy between measurable decay time $\tau_{m}(t \rightarrow 0)$ and actual lifetime depending on both the bias light parameter $\xi$ and the injection dependence represented by $d \tau /\left.d \Delta n\right|_{\Delta n_{0}}$. It confirms previous statements about the discrepancy's dependence on $d \tau /\left.d \Delta n\right|_{\Delta n_{0}}$. Moreover, it provides accurate quantitative information appropriate for a determination of actual lifetime from measured decay times.

\section{A. Discussion of limiting cases}

It appears worthwhile to shed light on the limiting cases of Eq. (14): In the trivial case of $d \tau /\left.d \Delta n\right|_{\Delta n_{0}}=0$, there is evidently no mismatch between $\tau_{m}(t \rightarrow 0)$ and actual lifetime.

The case $\xi=0$ represents zero bias light. Here, the injection dependence of lifetime does not induce a mismatch between $\tau_{m}(t \rightarrow 0)$ and lifetime. A transient injectiondependent measurement of decay time $e^{9,10}$

$$
\tau_{m}(\Delta n)=-\frac{\Delta n}{d \Delta n / d t}
$$

after an abrupt and-most importantly-complete switch-off of generation rate (i.e., $\xi=0$ ), therefore, directly yields actual lifetime. This particularly applies to the widespread lifetime analysis on the basis of transient or quasi-transient photoconductance. ${ }^{11,12}$ It follows from Eq. (14) that for non-vanishing $d \tau /\left.d \Delta n\right|_{\Delta n_{0}}$ the derivative of $\tau_{m}(t \rightarrow 0)$ with respect to $\xi$ at $\xi=0$ is also non-vanishing. Therefore, it must be emphasized that even a very faint measurable bias light $(\xi \neq 0)$ is expected to induce a measurable mismatch between $\tau_{m}(t \rightarrow 0)$ and carrier lifetime, as also visualized in Fig. 1.

The remaining limiting case to be discussed is the case of $\xi \rightarrow 1$. This is the case of an infinitesimal change of generation rate. It is also the idealized case of a differential decay time measurement. For $\xi \rightarrow 1$, Eq. (14) clearly indicates a maximal effect of the injection dependence of lifetime on the mismatch between $\tau_{m}(t \rightarrow 0)$ and actual lifetime (cf. Fig. 1). It appears worth noting that the expression of Eq. (14) is equal to the term $U^{-1}$ of Eq. (6). This is seized in the treatment of time-modulated experiments in the following section.

Due to the structure of Eq. (14), the mismatch by which $\tau_{m}(t \rightarrow 0)$ differs from lifetime is always more pronounced for a positive derivative of lifetime with respect to excess carrier density than for a negative derivative of equal absolute value. ${ }^{13}$ This is exemplified in Fig. 1, and it also appears throughout the simulation results depicted in Figs. 2-5.

\section{B. Relation to time-modulated measurements}

In the following, it is shown that the measurement of the phase shift between quasi-steady-state harmonic excess

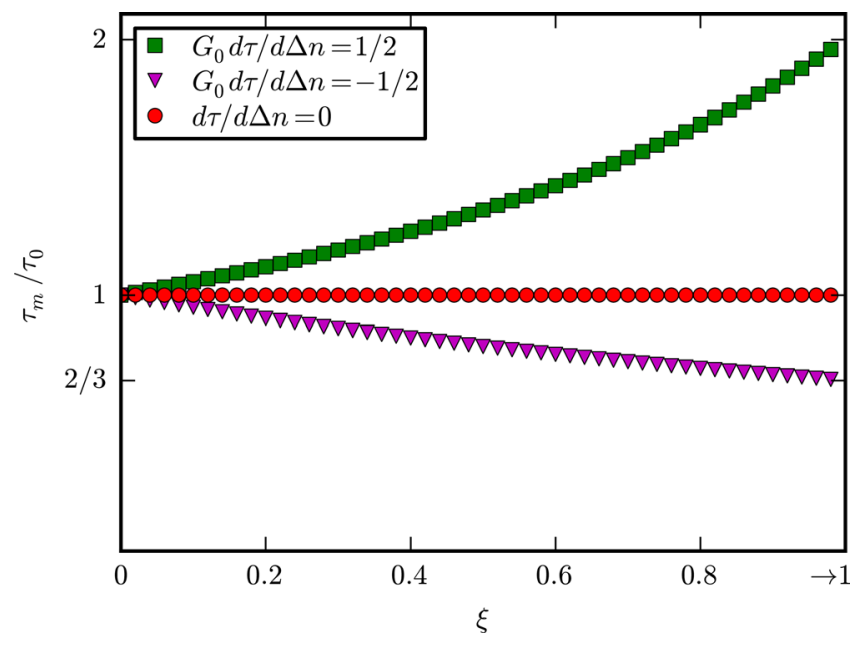

FIG. 1. Illustration of the discrepancy (ratio) between a measurable decay time $\tau_{m}$ and actual lifetime $\tau_{0}$ as a function of a bias light parameter $\xi<1$ with $G(t \leq 0)=G_{0}$ and $G(t>0)=\xi G_{0}$. Three situations are plotted: a positive derivative of lifetime with respect to excess carrier density with $G_{0} d \tau / d \Delta n=0.5$, a negative derivative with $G_{0} d \tau / d \Delta n=-0.5$, and an injection-independent lifetime. In any case, the discrepancy $\tau_{m} / \tau$ is most pronounced in the limit $\xi \rightarrow 1$. 
carrier generation $G(t)$ and excess carrier density $\Delta n(t)$ is in the end a measurement of decay time $\tau_{m}$.

For injection-independent lifetime, the phase shift is equal to lifetime. ${ }^{8}$ This can be seen from the solution of Eq. (4) for a harmonically oscillating excess carrier generation rate $G(t)=G_{0} \exp i \omega t$, which reads

$$
\Delta n(t)=\frac{G_{0}}{i \omega+1 / \tau} \exp i \omega t .
$$

The first order Taylor series expansions $(i \omega+1 / \tau)^{-1}$ $\approx \tau(1-i \omega \tau) \approx \tau \exp (-i \omega \tau)$ are very accurate if a quasisteady-state condition $\omega \ll \tau^{-1}$ is satisfied. This implies a phase lag $\tau$ between excess carrier generation and excess carrier density. However, for the general case of injectiondependent lifetime this is not true, because the phase shift corresponds to $\tau_{m}$ rather than $\tau$. For a rigorous proof of this statement, let us draw on Eq. (6), which is the linearization of the continuity equation, incorporating the injection dependence of carrier lifetime. Other than for the step-type excess carrier generation underlying Eq. (6), let us now assume an additional inhomogeneity of the form $G(t)=G_{0}+G_{1} \exp i \omega t$, featuring a sufficiently faint amplitude $G_{1}$ in order to ensure accuracy of the linearization in Eq. (6) (for infinitesimal $G_{1}$, Eq. (6) would be exact). Therefore, the total inhomogeneity is $V+G(t)$, here. The solution is a superposition of the solutions to the inhomogeneities $V+G_{0}$ (cf. Eq. (8)) and $G_{1}$ expi $i \omega t$. With an initial value $\Delta n(t=0)=G_{0} \tau_{0}$, it reads

$$
\Delta n(t)=G_{0}\left(\tau_{0}+\frac{1}{i \omega+U} \exp i \omega t\right) .
$$

Here, the Taylor series expansions below Eq. (16) yield a quasi-steady-state phase lag $U^{-1}$, which corresponds to decay time $\tau_{m}$ in the limit $\xi \rightarrow 1$ (cf. Eq. (14)) $\square$. Note that the constant bias generation $G_{0}$ is not needed for the mismatch between $\tau_{m}$ and actual carrier lifetime to occur here. It enables solely the use of the linearized Eq. (6) at a finite generation rate.

A harmonically time-modulated phase-sensitive lifetime approach $^{7,8,13-17}$ yields phase shifts corresponding to differential rather than actual lifetimes, irrespective of the existence of an additional steady-state bias light. In fact, a continuously time-modulated irradiation is the only true representative of the differential limit $\xi \rightarrow 1$, whereas a conventional light-biased decay time measurement ${ }^{2}$ is always restricted to finite differences between $\xi$ and 1 due to noise. The interpretation of harmonic time modulation as a lightbiased time sequence of infinitesimal changes of generation rate provides a descriptive understanding of the identity of decay time and phase shift. Changes of excess carrier generation rate induce relaxation of excess carrier density at a decay time $\tau_{m}$. The superposition of such relaxation processes brings about a phase lag $\tau_{m}$ between excess carrier generation rate and excess carrier density.

Quantitative experimental data on the mismatch between differential and actual lifetime in the limit $\xi \rightarrow 1$ have been published before. In 1999, $\mathrm{Schmidt}^{4}$ investigated the mismatch between differential lifetime as inferred from modulated free carrier absorption ${ }^{14-16}$ and actual lifetime as inferred from quasi-steady-state photoconductance ${ }^{11}$ —in an injection regime dominated by Auger recombination: with a mismatch between differential and actual lifetime by a factor of 3, excellent agreement between Schmidt's theoretical prediction for the Auger limit and experimental data was found. Note that the theory of light-biased decay time derived here predicts the same factor in the Auger limit for $\xi \rightarrow 1$ : with an actual Auger lifetime $\tau=a \Delta n^{-2}$, it follows that $G_{0}=\Delta n_{0} / \tau_{0}=\Delta n_{0}^{3} / a$ and $d \tau /\left.d \Delta n\right|_{\Delta n_{0}}=-2 a \Delta n_{0}^{-3}$. Insertion into Eq. (14) yields $\tau_{0}=3 \tau_{m}(t \rightarrow 0)$.

The determination of injection-dependent lifetime via the self-consistent solution of the continuity equation ${ }^{6}$ is generally unaffected by the mismatch between $\tau_{m}(t \rightarrow 0)$ and lifetime. This is due to the fact that if self-consistency is satisfied, the continuity equation is solved in a self-contained manner, which also takes injection dependence of lifetime into account. This is also demonstrated in self-consistent lifetime analyses of simulated excess carrier density for injection-dependent lifetime curves shown in Figs. 5 and 6.

\section{Interpretation of differential lifetime}

Equation (11) explicitly reveals the differential nature of the decay time $\tau_{m}(t \rightarrow 0)$, which is a change of excess carrier density per change of generation rate. The perspective of Eq. (11) provides a very descriptive insight into the origin of the discrepancy between differential and actual lifetime. With both steady-state excess carrier density $\Delta n=G \tau$ and an injection-dependent lifetime $d \tau / d \Delta n \neq 0$, the relative change of excess carrier density cannot equal the relative change of generation rate. In the case of $d \tau / d \Delta n>0$, the relative change of excess carrier density must be greater than the relative change of generation rate, and vice versa.

According to Eq. (11), the measurable decay time in the limit $\xi \rightarrow 1$ becomes

$$
\lim _{\xi \rightarrow 1} \tau_{m}(t \rightarrow 0)=\frac{d \Delta n}{d G} .
$$

Analogous to derivations by Aberle et $a l .^{2}$ and Schuurmans et $a l .,^{3}$ injection-dependent actual lifetime is

$$
\tau\left(\Delta n_{0}=G_{0} \tau\right)=\lim _{\substack{\xi \rightarrow 1 \\ t \rightarrow 0}} \frac{1}{G_{0}} \int_{0}^{G_{0}} d G \cdot \tau_{m}(G) .
$$

Historically, measurement and integration of the decay time $\tau_{m}(G)$ over all generation rates $G \leq G_{0}$ were found inevitable for a correct determination of actual from differential recombination properties. In fact, such measurements are time-consuming and additionally complicated by limitations at $G \rightarrow 0$ due to the instrumentation's sensitivity limit. ${ }^{2}$

In contrast, Eq. (14) reveals a way to correctly determine actual lifetime from a combination of at least two measurements of $\tau_{m}(G)$ to be conducted at adjacent generation rates. In the differential limit $\xi \rightarrow 1$, and with an index $i$ denoting each measurement, Eq. (14) reads

$$
\tau_{i}\left(G_{i}\right)=\tau_{m}\left(G_{i}\right) \cdot\left(1-\left.G_{i} \frac{d \tau}{d \Delta n}\right|_{\Delta n_{i}}\right) .
$$


The quantity to be determined is the actual (true) injectiondependent lifetime $\tau_{i}\left(G_{i}\right)$, while $G_{i}$ and $\tau_{m}\left(G_{i}\right)$ are directly measurable. Presuming local linearity of carrier lifetime $\tau(\Delta n)$ at the generation rates of interest-i.e., presuming locally constant $d \tau /\left.d \Delta n\right|_{\Delta n_{i}}$-the lifetimes $\tau_{2}\left(\tau_{1}\right)$ can be expressed as functions of each other via Eq. (5). Therefore, both $\tau_{i}\left(G_{i}\right)$ and $d \tau /\left.d \Delta n\right|_{\Delta n_{i}}$ can be determined from two measurements of $\tau_{m}\left(G_{i}\right)$. Even though the assumption of local linearity of $\tau(\Delta n)$ may be violated, Eq. (20) yet allows iterative determination of $\tau_{i}\left(G_{i}\right)$ from a set of more than two measured decay times $\tau_{m}\left(G_{i}\right)$ at generation rates $G_{i}$.

We have proposed a related approach to determine injection-dependent lifetime from measured phase shifts (based on time-modulated photoluminescence) in the past. $^{7,8,13}$ Originally, this so-called self-sufficient approach $^{7}$ determined carrier lifetime from measured phase shifts via numeric solution of the continuity equation. With the theory of light-biased decay time derived herein, the above numeric procedure can be replaced by a very fast analytic calculation on the basis of Eq. (20) - thereby accelerating the self-sufficient approach by more than two orders of magnitude in terms of computing time. In order to clearly denote this advanced analytic self-sufficient lifetime analysis, it shall be referred to as a differential-to-actual ( $d 2 a$ ) lifetime analysis in the following. The data shown in Figs. 5 and 6 confirm that the $d 2 a$ analysis reliably enables determination of actual lifetime from differential lifetime.

Note that harmonically time-modulated dynamic lock-in techniques sensitive to the phase shift between irradiation and excess carrier density have been known and used for lifetime analyses before. ${ }^{14-17}$ The above sketched approach provides a path for these techniques to overcome their constraint to measurements of injection-independent lifetimes.

\section{VALIDATION: NUMERICAL SIMULATIONS}

In order to quantitatively validate the implications of the theory of light-biased decay time-and particularly the conclusions from Eq. (14) — we conducted finite element simulations of the solution of the time-dependent continuity equation (Eq. (4)). With a time index $i$, the discrete representation of Eq. (4) is

$$
\Delta n\left(t_{i+1}\right)=\Delta n\left(t_{i}\right)+\left(t_{i+1}-t_{i}\right)\left(G\left(t_{i}\right)-\frac{\Delta n\left(t_{i}\right)}{\tau\left(\Delta n\left(t_{i}\right)\right)}\right) .
$$

The essential advantage of this representation is that it enables computation of excess carrier density $\Delta n(t)$ for nearly any form of $G(t)$ and $\tau(\Delta n)$. For sufficiently small time intervals $t_{i+1}-t_{i} \ll \tau$, it yields a virtually exact solution of the time-dependent continuity equation.

Our simulation covers two different scenarios concerning the time evolution of the generation rate: scenario A represents an abrupt change of generation rate from an initial steady state generation rate $G_{0}$ to a generation rate $\xi G_{0}$ with $0 \leq \xi<1$ (cf. Fig. 2). The initial generation rate is set to $G_{0}=10^{19} \mathrm{~cm}^{-3} \mathrm{~s}^{-1}$. Scenario B represents a sinusoidally time-modulated generation rate at a modulation time much greater than lifetime (quasi-steady-state)—likewise peaking at $G_{0}=10^{19} \mathrm{~cm}^{-3} \mathrm{~s}^{-1}$ (cf. Fig. 4). Further, our simulation distinguishes between three different scenarios concerning the injection dependence of lifetime $\tau(\Delta n)$ : scenario I represents a linearly increasing lifetime curve with a slope $d \tau / d \Delta n=5 \times 10^{-20} \mathrm{~cm}^{3} \mathrm{~s}$ and an intercept $\tau(0)=5 \times 10^{-4} \mathrm{~s}$. Scenario II represents a linearly decreasing lifetime curve with a slope $d \tau / d \Delta n=-5 \times 10^{-20} \mathrm{~cm}^{3} \mathrm{~s}$ and an intercept $\tau(0)=1.5 \times 10^{-3} \mathrm{~s}$. Scenario III finally represents an injection-independent lifetime curve $\tau=10^{-3} \mathrm{~s}$. All three scenarios intersect at $\tau\left(\Delta n=10^{16} \mathrm{~cm}^{-3}\right)=10^{-3} \mathrm{~s}$, respectively. With the initial (A) or maximum (B) generation rates as specified above, the initial (A) or maximum (B) excess carrier density is in any case $\Delta n_{0}=10^{16} \mathrm{~cm}^{-3}$.

\section{A. Abrupt change of generation rate}

For an abruptly changing generation rate, we modelled $\Delta n(t)$ for three different values of the bias light parameter $\xi=G / G_{0}$, involving $\xi=0.999$ as a value representative of the limit $\xi \rightarrow 1, \xi=0.5$ as an intermediate value, and $\xi=0$ for the case without bias light. Injection-dependent decay time was determined as

$$
\tau_{m}(\Delta n(t))=\frac{\Delta n(t)-\Delta n(t \rightarrow \infty)}{d \Delta n(t) / d t}
$$

for any of the scenarios I-III. Fig. 2 depicts excess carrier density as a function of time for all lifetime scenarios and for all three values of $\xi$. In the case of injection-dependent lifetime and $\xi>0$, the relative change of excess carrier density differs from the relative change of generation rate. Fig. 2 quantitatively motivates the difference between relative changes of excess carrier density and generation rate as the origin of the discrepancy between actual and differential lifetime. In the limit $\xi \rightarrow 1$ (cf. Fig. 2(a)), a value of $G_{0} d \tau / d \Delta n= \pm 0.5$ leads to a factor between relative changes of excess carrier density and generation rate of $2(+)$ or $2 / 3(-)$. In the intermediate case $\xi=0.5$ (cf. Fig. 2(b)), these factors are $4 / 3(+)$ or $4 / 5(-)$, and for $\xi=0$ (cf. Fig. 2(c)) they are both unity. Note the quantitative analogy to the mismatch between decay time and lifetime shown in Fig. 3.

Fig. 3 compares actual injection-dependent (true) lifetime to decay time $\tau_{m}(\Delta n(t))$ according to Eq. (22). As can be seen in plots (a) and (b), for $\xi>0$ the decay time measurement cannot cover the entire range of excess carrier density. As expected, for $\xi>0$ a positive derivative of lifetime with respect to excess carrier density leads to $\tau_{m} / \tau>1$, whereas a negative derivative leads to $\tau_{m} / \tau<1$. The greater $\xi$, the greater is the discrepancy between decay time and lifetime. Beyond that, every aspect of Eq. (14) is also quantitatively substantiated by this simulation result: with $G_{0} d \tau / d \Delta n=0.5$, Eq. (14) predicts $\tau_{m}(\xi \rightarrow 1) / \tau=2$ and $\tau_{m}(\xi=0.5) / \tau=4 / 3$, whereas with $G_{0} d \tau / d \Delta n=-0.5, \quad \tau_{m}(\xi \rightarrow 1) / \tau=2 / 3$ and $\tau_{m}(\xi=0.5) / \tau=4 / 5$ are predicted. For $\xi=0$, the simulation result confirms that decay time measurements without bias light $^{9-12}$ coincide with actual lifetimes.

\section{B. Harmonic time modulation of generation rate}

Fig. 4 depicts both a sinusoidal time modulation of optical irradiation of a silicon substrate (at a maximum generation rate 
(a) $\xi=G / G_{0}=0.999$

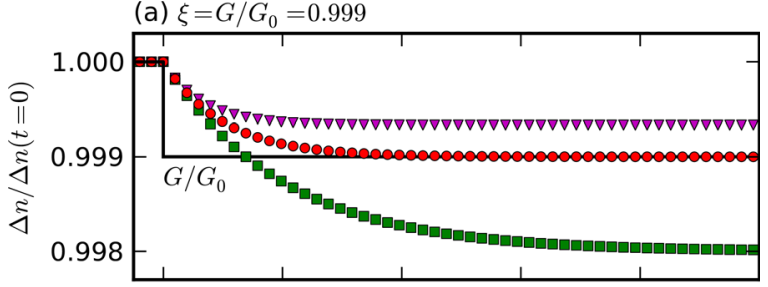

(b) $\xi=G / G_{0}=0.5$

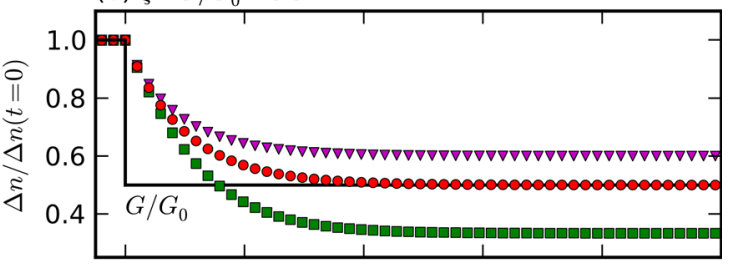

(c) $\xi=G / G_{0}=0$

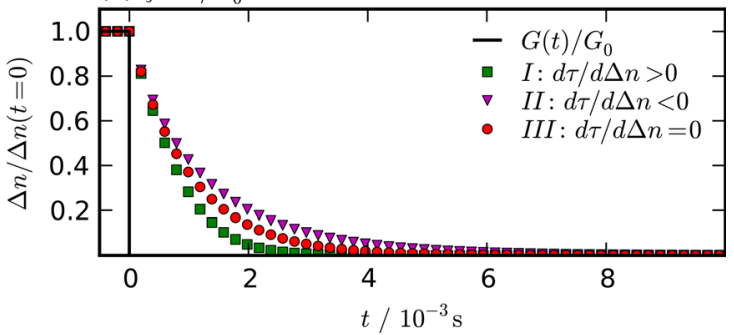

FIG. 2. Depiction of excess carrier density as a function of time after an abrupt change of generation rate at $t=0$. Here, three scenarios of $\tau(\Delta n)$ are discussed, the actual lifetime curves (as used for the simulation of $\Delta n(t)$ ) are depicted in Fig. 3 as solid lines, respectively. The relative change of excess carrier density is greater than the relative change of generation rate if $d \tau / d \Delta n>0$, and vice versa. This gives rise to a discrepancy between decay time and lifetime for light-biased measurements $(\xi>0)$, as can be seen in Fig. 3. The resulting curves also quantitatively confirm the validity of Eq. (14) (cf. Fig. 1).

of $G_{0}=10^{19} \mathrm{~cm}^{-3} \mathrm{~s}^{-1}$ and at a modulation frequency of $3.42 \mathrm{~s}^{-1}$ ) as well as the modelled quasi-steady-state excess carrier density of the substrate for the above mentioned linear curve shapes I-III of the substrate's lifetime $\tau(\Delta n)$. From the plot of a total modulation cycle (a), it can be seen that the injection dependence has a crucial impact on the relative curve shapes of excess carrier density. A positive derivative of lifetime with respect to excess carrier density enhances the absolute value of the peak curvature of excess carrier density, whereas a negative derivative lowers the absolute value of the peak curvature. These curvatures already bear information about the origin of the discrepancy between actual and differential lifetime, being the ratio between the relative change of excess carrier density and the relative change of generation rate (cf. Eq. (11)). In the detailed plot of the curve peaks (b), phase shifts between maxima of generation rate and excess carrier density are highlighted. Most prominently, these phase shifts exactly coincide with predictions of decay time according to Eq. (14) in the limit $\xi \rightarrow 1$. Although all three lifetime scenarios feature a peak lifetime of $\tau\left(\Delta n_{0}=10^{16} \mathrm{~cm}^{-3}\right)=10^{-3} \mathrm{~s}$, the phase shifts are $\tau_{m}\left(\Delta n_{0}\right)=2 \times 10^{-3} \mathrm{~s}$ for $G_{0} d \tau / d \Delta n=0.5$ (I) and $\tau_{m}\left(\Delta n_{0}\right)=$ $2 / 3 \times 10^{-3}$ s for $G_{0} d \tau / d \Delta n=-0.5$ (II).

In Fig. 5, phase shifts between generation rate and modelled excess carrier density were determined not only at maximal signals but also at lower generation rates. The details of this calculation are explained elsewhere. ${ }^{7}$ Comparison of this injection-dependent phase shift to actual injection-dependent
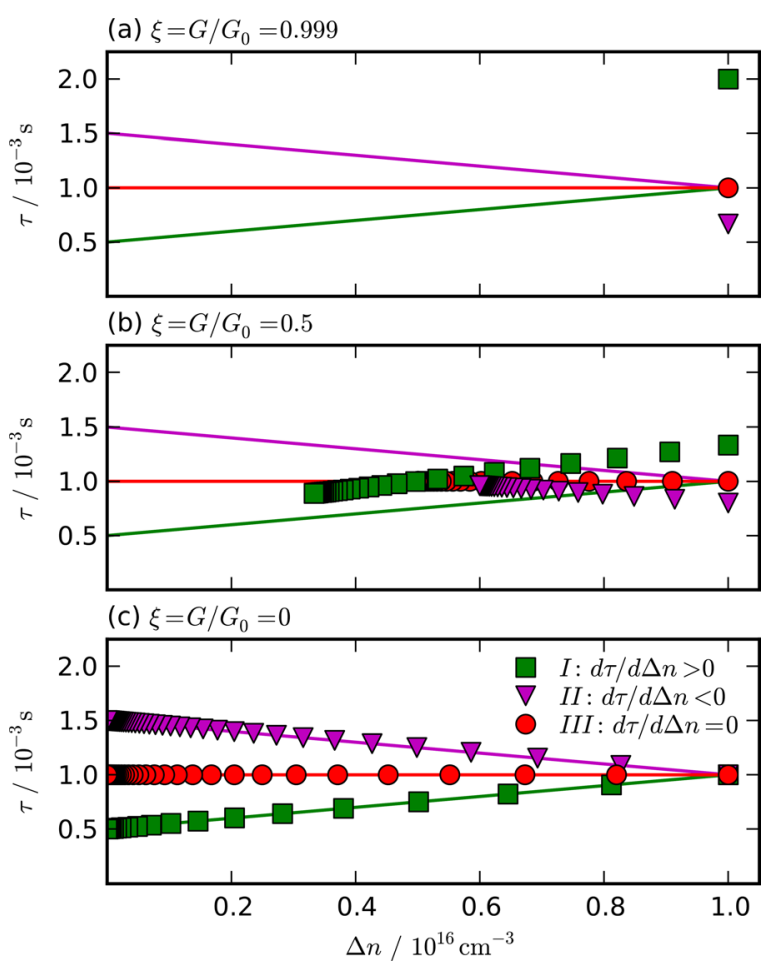

FIG. 3. Comparison of measurable decay time $\tau_{m}$ according to Eq. (22) (symbols) and actual lifetime (solid lines) for the scenarios of an abrupt change of generation rate at $t=0$ as shown in Fig. 2. All results quantitatively agree with Eq. (14). For $\xi=0$, decay time and actual lifetime coincide. For $\xi>0$, decay time is greater than actual lifetime if $d \tau / d \Delta n>0$ and smaller than actual lifetime if $d \tau / d \Delta n<0$. The greater $\xi$, the greater is the discrepancy between decay time and actual lifetime (cf. Fig. 1).

(true) lifetime again confirms Eq. (14): with a constant value of $d \tau / d \Delta n$ the ratio between decay time (phase shift) and actual lifetime approaches unity when approaching very small generation rates $G \rightarrow 0$. We also performed two dynamic lifetime analyses on the basis of the simulated data shown in Fig. 4: a self-consistent solution of the continuity equation, ${ }^{6}$ and a phase-sensitive $d 2 a$ (differential-to-actual) lifetime analysis based on the theory of light-biased decay time derived here (cf. Eq. (20)). Both analyses yield true lifetimes for any of the discussed scenarios I-III. This demonstrates both the general validity of the self-consistent approach for injection-dependent lifetime measurements, and the possibility to correctly determine actual lifetime from differential lifetime measurements via the $d 2 a$ technique without integration over the entire injection range.

The simulation results presented so far were deliberately based on linear lifetime curves $\tau(\Delta n)$ in order to instructively point out their quantitative agreement with Eq. (14). As previously indicated though, the determination of actual injection-dependent carrier lifetime from decay time measurements via Eq. (14) is by no means restricted to linear lifetime curves, rather it is generally valid. This is demonstrated in Fig. 6, where the same analyses as of Fig. 5 are applied to simulated dynamic photoluminescence measurements in the injection ranges of both the Shockley-Read-Hall ascent and the Auger descent of a typical injection-dependent lifetime curve. Despite the fact that this lifetime curve features a 

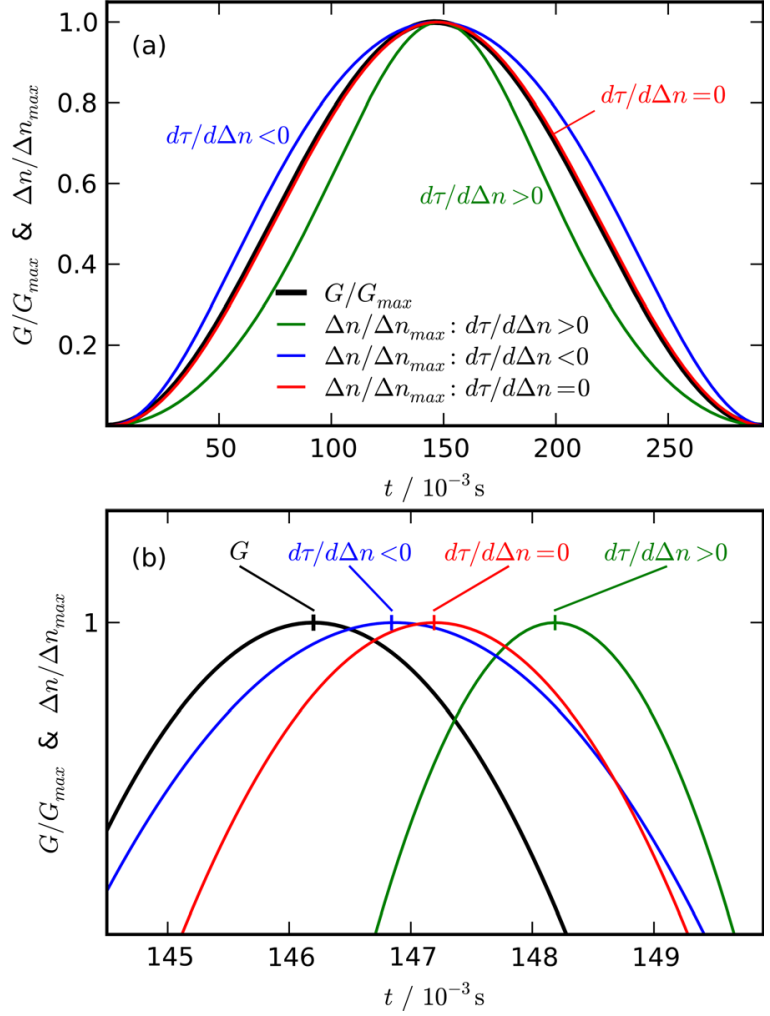

FIG. 4. Depiction of relative generation rate and excess carrier density in the case of a harmonically time-modulated irradiation. The excess carrier density curves shown here represent numeric solutions of the timedependent continuity equation for different linear curve shapes of $\tau(\Delta n)$. Three linear lifetime scenarios are plotted: a positive derivative of lifetime with respect to excess carrier density $d \tau / d \Delta n=5 \times 10^{-20} \mathrm{~cm}^{3} \mathrm{~s}$, a negative derivative $d \tau / d \Delta n=-5 \times 10^{-20} \mathrm{~cm}^{3} \mathrm{~s}$, and a constant lifetime of $\tau=10^{-3} \mathrm{~s}$. All lifetime curves intersect at $\tau\left(\Delta n=10^{16} \mathrm{~cm}^{-3}\right)=10^{-3} \mathrm{~s}$, which also corresponds to the maximally achieved excess carrier density in each scenario (due to $G_{\max }=10^{19} \mathrm{~cm}^{-3} \mathrm{~s}^{-1}$ ). The upper plot (a) depicts a total modulation while the lower plot (b) focuses on the most relevant peak area. Plot (b) shows that phase shifts can exactly be predicted by Eq. (14) in the limit $\xi \rightarrow 1$.

pronounced curvature in the targeted injection ranges, both the self-consistent approach as well as the $d 2 a$ analysis yield actual effective carrier lifetime.

\section{CONCLUSIONS}

In this paper, it was pointed out that the origin of the discrepancy between light-biased decay time and actual lifetime is the difference between a relative change of generation rate and a corresponding relative change of excess carrier density. On this basis, we developed a theory of light-biased decay time. This theory provides a generally valid quantitative explanation of the discrepancy between light-biased decay time and actual lifetime. In practice, it enables the determination of actual lifetime from decay time measurements without the need of integration over the entire injection range.

The essential equation of the theory of light-biased decay time quantitatively describes the relation between actual lifetime and the directly measurable differential lifetime as a function of the product of two quantities: the first is the derivative of lifetime with respect to excess carrier
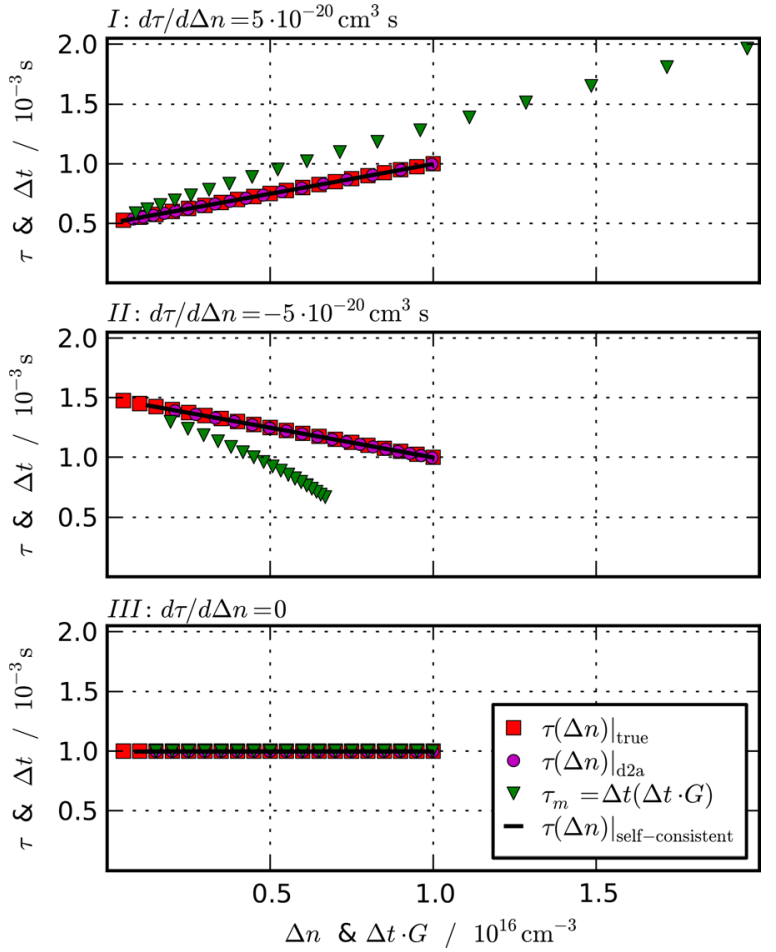

FIG. 5. Injection-dependent phase shifts $\tau_{m}=\Delta t(\Delta t \cdot G)$ between harmonically time-modulated generation rate and excess carrier density were compared to true injection-dependent lifetime for the three linear scenarios of $\tau(\Delta n)$ discussed here. All results of $\tau_{m}=\Delta t(\Delta t \cdot G)$ clearly confirm Eq. (14) in the limit $\xi \rightarrow 1$. Additionally, two injection-dependent lifetime analyses were performed - a self-consistent lifetime analysis ${ }^{6}$ and an advanced selfsufficient analysis-referred to as differential-to-actual $(d 2 a)$ lifetime analysis here. This analysis applies the theory of light-biased decay time (Eq. (20)) to injection-dependent phase shifts. ${ }^{7}$ Both analyses accurately yield true injection-dependent lifetime in any of the discussed scenarios. Therefore, integration over the entire injection range is not necessary in order to correctly determine injection-dependent lifetimes from measurements of decay time (or phase shift).

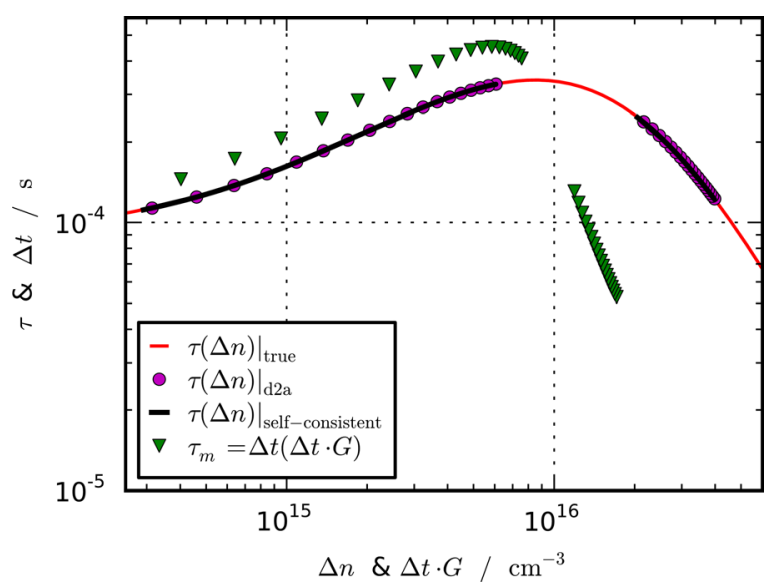

FIG. 6. Demonstration of the applicability of both the self-consistent ${ }^{6}$ and the differential-to-actual $(d 2 a)$ lifetime analyses to a nonlinear lifetime curve $\tau(\Delta n)$ : Self-consistent and $d 2 a$ lifetime analyses were performed on simulated dynamic photoluminescence measurements on the basis of the true carrier lifetime curve shown in the plot. Two measurements were simulated at injection conditions such that they covered the Shockley-Read-Hall ascent and the Auger descent of carrier lifetime, respectively. It can be seen that phase shifts $\tau_{m}=\Delta t(\Delta t \cdot G)$ substantially differ from actual carrier lifetime. Despite the nonlinearity of $\tau(\Delta n)$, both lifetime analyses accurately yield true injection-dependent effective carrier lifetime. 
density, and the second is a bias light parameter denoting the relative change of generation rate. At a finite derivative of lifetime with respect to excess carrier density, the mismatch between decay time and actual lifetime is most pronounced for infinitesimal changes of generation rate, whereas a complete switch-off of generation rate (no bias light) leads to identity of differential and actual lifetime.

Any decay time measurement featuring bias light is affected by a mismatch between the measurable differential decay time and actual lifetime. This involves both conventional truly light-biased transient decay time measurements ${ }^{1-4}$ as well as measurements featuring a continuous or even harmonic time modulation of irradiation, which directly exploit the phase shift between irradiation and excess carrier density for the determination of lifetime. ${ }^{14-17}$ In the latter case, continuous time modulation can be interpreted as a light-biased time sequence of infinitesimal changes of generation rate. Therefore, these techniques represent the differential limit of the theory of light-biased decay time derived here. Yet, not all time-modulated lifetime techniques are sensitive to differential lifetime-e.g., the self-consistent solution of the time-dependent continuity equation ${ }^{6}$ directly yields actual lifetime.

We had previously outlined ways to numerically obtain actual lifetime from phase sensitive (i.e., differential) lifetime techniques $^{7,8,13}$ —elsewhere referred to as self-sufficient due to a minimum of additional information required for a lifetime determination. With the theory of light-biased decay time presented herein, the self-sufficient approach was transformed from a numerical foundation to an analytical one- - henceforth referred to as differential-to-actual ( $d 2 a$ ) lifetime analysis.

\section{ACKNOWLEDGMENTS}

This work was funded by the Fraunhofer Gesellschaft within the project Silicon BEACON.

\section{APPENDIX: VALIDITY OF THEORY FOR NONLINEAR LIFETIMES}

In the following, a proof of the general validity of Eq. (13) in the limit $\xi \rightarrow 1$ is given. Statement: The curvature of any nonlinear effective carrier lifetime $\tau(\Delta n)$-represented by its second derivative with respect to excess carrier density-does not affect $\Delta n_{\infty}=\Delta n(t \rightarrow \infty)$ in the limit $\xi \rightarrow 1$ (e.g., harmonic time modulation of excess carrier generation).

Proof: With $\Delta n_{0}=\Delta n(t=0)=\tau_{0} G_{0}, d_{1}=d \tau /\left.d \Delta n\right|_{\Delta n_{0}}$, and $d_{2}=d^{2} \tau /\left.d \Delta n^{2}\right|_{\Delta n_{0}}$, the second order Taylor series expansion of lifetime reads

$$
\begin{aligned}
\tau\left(\Delta n_{\infty}\right)= & \tau_{0}+\left(\Delta n_{\infty}-\Delta n_{0}\right) d_{1}+\frac{1}{2}\left(\Delta n_{\infty}-\Delta n_{0}\right)^{2} d_{2} \\
& +O\left(\left(\Delta n_{\infty}-\Delta n_{0}\right)^{3}\right) .
\end{aligned}
$$

Insertion of this expression into Eq. (12) yields

$$
\Delta n_{\infty}=\xi G_{0} \cdot\left(\tau_{0}+\left(\Delta n_{\infty}-\Delta n_{0}\right) d_{1}+\frac{1}{2}\left(\Delta n_{\infty}-\Delta n_{0}\right)^{2} d_{2}\right)
$$

The positive solution of this quadratic equation in $\Delta n_{\infty}$ is

$$
\Delta n_{\infty}=\frac{1-\xi G_{0} d_{1}+\xi G_{0} \Delta n_{0} d_{2}+\sqrt{\left(\xi G_{0} d_{1}-1\right)^{2}+2 \xi G_{0} \Delta n_{0} d_{2} \cdot(1-\xi)}}{\xi G_{0} d_{2}} .
$$

Let the square root term in the above equation be denoted $g(1-\xi)$. The first order Taylor series expansion of this function is

$g(1-\xi)=\xi G_{0} d_{1}-1+\frac{\xi G_{0} \Delta n_{0} d_{2}}{\xi G_{0} d_{1}-1} \cdot(1-\xi)+O\left((1-\xi)^{2}\right)$.

In the limit $\xi \rightarrow 1$, the above Taylor expansion is exact, so Eq. (A3) reads

$$
\lim _{\xi \rightarrow 1} \Delta n_{\infty}=\xi \Delta n_{0} \frac{1-G_{0} d_{1}}{1-\xi G_{0} d_{1}} .
$$

This expression is independent of the curvature $d_{2}$ and therefore identical to Eq. (13) $\square$.

\footnotetext{
${ }^{1}$ R. Brendel, Appl. Phys. A 60, 523 (1995).

${ }^{2}$ A. G. Aberle, J. Schmidt, and R. Brendel, J. Appl. Phys. 79, 1491 (1996).

${ }^{3}$ F. M. Schuurmans, A. Schönecker, A. R. Burgers, and W. C. Sinke, Appl.

Phys. Lett. 71, 1795 (1997).

${ }^{4}$ J. Schmidt, IEEE Trans. Electron Devices 46, 2018 (1999).

${ }^{5}$ R. A. Sinton and T. Trupke, Prog. Photovoltaics 20, 246 (2012).
}

${ }^{6}$ T. Trupke, R. A. Bardos, and M. D. Abbott, Appl. Phys. Lett. 87, 184102 (2005).

${ }^{7}$ J. A. Giesecke, M. C. Schubert, and W. Warta, Phys. Status Solidi A 209, 2286 (2012).

${ }^{8}$ J. A. Giesecke, M. C. Schubert, D. Walter, and W. Warta, Appl. Phys. Lett. 97, 092109 (2010).

${ }^{9}$ H. W. Curtis and R. L. Verkuil, in Lifetime Factors in Silicon, San Diego 15-16 February 1979, edited by R. D. Westbrook, ASTM Special Technical Publication No. 712 (American Society for Testing and Materials, Philadelphia, 1980), pp. 210-224.

${ }^{10}$ D. E. Kane and R. M. Swanson, in Proceedings of the 18th IEEE PVSC, Las Vegas (1985), pp. 578-583.

${ }^{11}$ R. A. Sinton and A. Cuevas, Appl. Phys. Lett. 69, 2510 (1996).

${ }^{12}$ H. Nagel, C. Berge, and A. G. Aberle, J. Appl. Phys. 86, 6218 (1999).

${ }^{13}$ J. A. Giesecke, D. Walter, F. Kopp, P. Rosenits, M. C. Schubert, and W. Warta, in Proceedings of the 35th IEEE PVSC, Honolulu (2010), pp. 847851.

${ }^{14}$ F. Sanii, F. P. Giles, R. J. Schwartz, and J. L. Gray, Solid-State. Electron. 35, 311 (1992).

${ }^{15}$ S. W. Glunz, A. B. Sproul, W. Warta, and W. Wettling, J. Appl. Phys. 75, 1611 (1994).

${ }^{16}$ S. W. Glunz and W. Warta, J. Appl. Phys. 77, 3243 (1995).

${ }^{17}$ R. Brüggemann and S. Reynolds, J. Non-Cryst. Solids 352, 1888 (2006). 